\title{
On a Semi-Linear Wave Equation Associated with Memory Conditions at the Boundaries: Stability and Asymptotic Expansion
}

\author{
Út V. Lê
}

Communicated by S.-T. Yau, received August 25, 2008.

\begin{abstract}
In this paper the stability and asymptotic expansion of the weak solution of an initial-boundary problem relating to a semi-linear wave equation and two integral equations at the boundaries are given.
\end{abstract}

\section{CONTENTS}

1. Introduction 329

2. Preliminary results and notations 332

3. Unique solvability and regularity 334

4. The stability of the weak solution $\quad 335$

5. Low-frequency asymptotic expansion of the weak solution 339

6. Appendix: The detailed proof of Lemma $3 \quad 343$

References $\quad 346$

\section{Introduction}

We study the solution $u(x, t)$ of following semi-linear equation

$$
\begin{aligned}
\frac{\partial^{2} u}{\partial t^{2}}(x, t)-\frac{\partial}{\partial x}\left(\mu(x, t) \frac{\partial u}{\partial x}(x, t)\right) & +G(u(x, t)) \\
& +H\left(\frac{\partial u}{\partial t}(x, t)\right)=F(x, t)
\end{aligned}
$$

1991 Mathematics Subject Classification. 35L15, 35L20; 35L70.

Key words and phrases. Contracted procedure, semi-linear wave equation, stability, asymptotic expansion.

The author is currently supported by the Academy of Finland and Emil Aaltonen Foundation.

(C)2008 International Press 
where $0<x<1,0<t<T$, associated with initial-boundary values given by

$$
\begin{gathered}
\mu(0, t) \frac{\partial u}{\partial x}(0, t)=g_{0}(t)+\int_{0}^{t} k_{0}(t-s) u(0, s) d s \\
-\mu(1, t) \frac{\partial u}{\partial x}(1, t)=g_{1}(t)+\int_{0}^{t} k_{1}(t-s) u(1, s) d s, \\
u(x, 0)=u_{0}(x), \frac{\partial u}{\partial t}(x, 0)=u_{1}(x),
\end{gathered}
$$

where $\mu, G, H, F, g_{0}, k_{0}, g_{1}, k_{1}, u_{0}$ and $u_{1}$ are given real functions satisfying conditions specified later. The terms $G(u(x, t)), H\left(\frac{\partial u}{\partial t}(x, t)\right)$ are, respectively, called the damping, the source term of Eq. (1.1), and we shortly call the sum $G(u(x, t))+H\left(\frac{\partial u}{\partial t}(x, t)\right)$ the damping-source term.

The problems of wave equations associated with memory conditions or integral equations at the boundaries have interested many mathematicians (see [5], [11], [14], [15], [19]-[25], [27]).

When $\mu(x, t) \equiv 1$ and $F(x, t)=0$, in [19], Nguyen and Alain considered problem $(1.1),(1.4)$ in the case of the full nonlinear damping-source term of $u$ and $\frac{\partial u}{\partial t}$ associated with the homogeneous boundary at $x=0$ and the non-homogeneous boundary condition at $x=1$ given by

$$
-\frac{\partial u}{\partial x}(1, t) \equiv Q(t)=h u(1, t)-g(t)-\int_{0}^{t} k(t-s) u(1, s) d s,
$$

where $h$ is a positive constant; $Q, g$ and $k$ are given functions. We note that (1.5) is deduced from a Cauchy problem for an ordinary differential equation at the boundary $x=1$ as follows

$$
\left\{\begin{array}{l}
Q^{\prime \prime}(t)+\omega^{2} Q(t)=h \frac{\partial^{2} u}{\partial t^{2}}(1, t), t \in(0, T), \\
Q(0)=Q_{0}, Q^{\prime}(0)=Q_{1}
\end{array}\right.
$$

where $\omega>0, Q_{0}$ and $Q_{1}$ are given constants. This problem is a mathematical model describing the shock of a rigid body and a nonlinear viscoelastic bar resting on a rigid base. In this article, the authors obtained the unique solvability of the weak solution.

In [25], Santos studied the asymptotic behavior of the solution of problem (1.1), (1.2), (1.4) in the case of $\mu(x, t) \equiv \mu(t), G(u)=H\left(\frac{\partial u}{\partial t}\right)=0, F(x, t)=0$ associated with a boundary condition of memory type at $x=1$ as follows

$$
u(1, t)+\int_{0}^{t} g(t-s) \mu(s) \frac{\partial u}{\partial x}(1, s) d s=0, t>0,
$$

in which $g$ and $\mu$ are given functions. It is noted that the boundary conditions (1.5) and (1.7) are similar since their formal differences can be crossed out after solving the Volterra equation with respect to the variable $u(1, t)$ given by $(1.7)$.

In $[\mathbf{2 1}, \mathbf{2 2}, \mathbf{2 3}]$, Nguyen, Lê and T. Nguyen considered the unique existence, stability, regularity in time variable and asymptotic expansion for the solution of problem (1.1)-(1.4) when $\mu(x, t) \equiv \mu(t), G(u)=K u, H\left(\frac{\partial u}{\partial t}\right)=\lambda \frac{\partial u}{\partial t}$ and the boundary condition (1.2) is homogeneous and the boundary value at $x=1,(1.3)$, is

$$
-\mu(t) \frac{\partial u}{\partial x}(1, t)=g(t)+K_{1}(t) u(1, t)+\lambda_{1}(t) \frac{\partial u}{\partial t}(1, t)+\int_{0}^{t} k(t-s) u(1, s) d s
$$


where $g, K_{1}, \lambda_{1}$ and $k$ are given functions.

When $\mu=1, G(u)=K|u|^{\alpha} u$ and $H\left(\frac{\partial u}{\partial t}\right)=\lambda\left|\frac{\partial u}{\partial t}\right|^{\beta} \frac{\partial u}{\partial t}$ for $K, \lambda, \alpha, \beta \geq 0$, Nguyen, Alain and Tran [20] studied the unique solvability, the regularity of the weak solution of problem (1.1), (1.4) associated with the boundary conditions as follows:

$$
\left\{\begin{array}{l}
\frac{\partial u}{\partial x}(0, t)=g(t)+h u(0, t)-\int_{0}^{t} k(t-s) u(0, s) d s, \\
\frac{\partial u}{\partial x}(1, t)+K_{1} u(1, t)+\lambda_{1} \frac{\partial u}{\partial t}(1, t)=0,
\end{array}\right.
$$

where $h, K_{1}, \lambda_{1}$ are given constants and $g, k$ are given functions. In the case of $\alpha=\beta=0$, the authors obtained the asymptotic expansion of the weak solution with respect to non-negative constants $K$ and $\lambda$.

In the above articles, the authors mainly applied Faedo-Galerkin approximation to study the unique solvability.

In the case of homogeneous boundaries, in [26], Sengul investigated the existence of the global attractor of Eq.(1.1) in the case of

$$
\mu(x, t)=1, H\left(\frac{\partial u}{\partial t}\right)=\alpha \frac{\partial u}{\partial t}, \alpha>0
$$

associated homogeneous boundary conditions and the initial conditions similar to (1.4). In [1], Aassila and Benaissa obtained the global unique solvability, also by Faedo-Galerkin approximation, and the decay for the solution of the following problem:

$$
\left\{\begin{array}{l}
\frac{\partial^{2} u}{\partial t^{2}}-\Phi\left(\int_{\Omega} \sum_{i=1}^{n}\left(\frac{\partial u}{\partial x_{i}}\right)^{2} d x\right) \sum_{i=1}^{n} \frac{\partial^{2} u}{\partial x_{i}^{2}} \\
\quad+g\left(\frac{\partial^{2} u}{\partial t^{2}}\right)+f(u)=0 \text { in } \Omega \times[0,+\infty[, \\
u(x, t)=0 \text { on } \Gamma \times[0,+\infty[, \\
u(x, 0)=u_{0}(x), \frac{\partial u}{\partial t}(x, 0)=u_{1}(x) \text { on } \Omega,
\end{array}\right.
$$

for $\Omega$ a bounded domain in $\mathbb{R}^{n}$ with a smooth boundary $\partial \Omega=\Gamma$, where $\Phi, g, f$, $u_{0}$ and $u_{1}$ are given functions. In [4], Benaddi and Rao obtained the energy decay rate of the solution by a shooting method for problem (1.1)-(1.4) where $\mu(x, t)=1$, $G$ and $H$ are linear, $F(x, t)=0$, and $g_{0}=g_{1}=k_{0}=k_{1} \equiv 0$ (or (1.2)-(1.3) are homogeneous). In addition, Phung [24] studied the stabilization of the wave equation with a localized linear dissipation in a three-dimensional bounded domain on which exists a trapped ray given by

$$
\left\{\begin{array}{l}
\frac{\partial^{2} u}{\partial t^{2}}-\sum_{i=1}^{3} \frac{\partial^{2} u}{\partial x_{i}^{2}}+\alpha(x) \frac{\partial u}{\partial t}=0 \text { in } \Omega \times \mathbb{R}^{+}, \\
u(x, t)=0 \text { on } \partial \Omega \times \mathbb{R}^{+}, \\
u(x, 0)=u_{0}, \frac{\partial u}{\partial t}(x, 0)=u_{1}(x) \text { in } \Omega,
\end{array}\right.
$$

for $\Omega$ a bounded domain in $\mathbb{R}^{3}$ with a boundary $\partial \Omega$ at least Lipschitz; $\alpha, u_{0}$ and $u_{1}$ are given functions.

Regarding Mikusiński calculus, D. Takači and A. Takači studied the existence of the solution of problem (1.1), (1.4) in the field of Mikusiński when $\mu(x, t)=1$, $G$ and $H$ are linear, and the boundary conditions are non-homogeneous.

In this paper, we study the stability and asymptotic expansion of the weak solution of problem (1.1)-(1.4). What we obtain here is considered as both the 
generalization and the more effective approach of those in Aassila and Benaissa [1], Nguyen and Alain [19], in Nguyen, Alain and Tran [20], in Santos [25], in Sengul [26], D. Takači and A. Takači $[\mathbf{2 7}]$ and in mine $[\mathbf{1 1}, \mathbf{1 4}, \mathbf{2 1}, \mathbf{2 2}, \mathbf{2 3}]$.

Furthermore, to obtain the unique solvability of problem (1.1)-(1.4), we apply a contracted procedure (see $[\mathbf{1 2}]$ and $[\mathbf{1 5}]$ ) which exceeds a routine application of usual methods, namely the Faedo-Galerkin method with the compactness argument and the monotone operator method, for semi-linear damped wave equations as popularized by Jacques-Louis Lions several years ago (see [16], [17]) and also by Songmu Zheng [30]. Moreover, by this contracted procedure, we can cover unsolvable cases related to problem (1.1)-(1.4) regarding the solvability, and if the solvability does not hold then we obviously fail to discuss neither the stability nor the asymptotic expansion involved in this paper.

\section{Preliminary results and notations}

First we introduce some preliminary results and notations used in this paper. We omit the definitions of usual function spaces: $C^{m}, L^{p}, W^{m, p}, H^{m}$ for $p \in[1,+\infty]$ and $m \in \mathbb{N}$.

We denote by $\langle\cdot, \cdot\rangle$ the scalar product in $L^{2}(0,1)$ or pair of dual scalar product of a continuous linear functional with an element of a function space. We denote by $\|\cdot\|_{X}$ the norm of a Banach space $X$ and by $X^{\prime}$ the dual space of $X$. We denote by $L^{p}(0, T ; X), 1 \leq p \leq \infty, T>0$, the Banach space of the real measurable functions $v:(0, T) \rightarrow X$, such that

$$
\|v\|_{L^{p}(0, T ; X)}=\left(\int_{0}^{T}\|v(t)\|_{X}^{p} d t\right)^{1 / p}<\infty \text { for } 1 \leq p<\infty
$$

and

$$
\|v\|_{L^{\infty}(0, T ; X)}=\operatorname{esssup}_{0<t<T}\|v(t)\|_{X} \text { for } p=\infty .
$$

In $H^{1}(0,1)$, we use the norm

$$
\|v\|_{H^{1}(0,1)}=\sqrt{\|v\|_{L^{2}(0,1)}^{2}+\left\|v^{\prime}\right\|_{L^{2}(0,1)}^{2}}, v \in H^{1}(0,1) .
$$

Then we have the following lemma whose proof is omitted:

LEMma 1. The embedding $H^{1}(0,1) \hookrightarrow C^{0}([0,1])$ is compact and

$$
\|v\|_{C^{0}([0,1])} \leq \sqrt{2}\|v\|_{H^{1}(0,1)}
$$

for all $v \in H^{1}(0,1)$.

Now let $x=\left(x_{1}, x_{2}\right), y=\left(y_{1}, y_{2}\right) \in \mathbb{Z}_{+}^{2}$, we denote

$$
\left\{\begin{array}{l}
y \leq x \Leftrightarrow\left\{\begin{array}{l}
y_{1} \leq x_{1}, \\
y_{2} \leq x_{2},
\end{array}\right. \\
x !=x_{1} ! x_{2} ! \\
C_{x}^{y}=\frac{x !}{y !(x-y) !} .
\end{array}\right.
$$

Then there is a lemma as follows: 
Lemma 2. Let $m \in \mathbb{Z}_{+}$and $n, i, j \in \mathbb{N}$. Then, the equality

$$
\left(\sum_{1 \leq i+j \leq n} a_{i j} \varepsilon^{i} \delta^{j}\right)^{m}=\sum_{m \leq i+j \leq m n}\left[a_{i j}\right]_{m} \varepsilon^{i} \delta^{j}
$$

holds for $\varepsilon, \delta, a_{i j} \in \mathbb{R}$ and

$$
\left[a_{i j}\right]_{m}=\left\{\begin{array}{l}
a_{i j}, i+j \leq n, m=1, \\
\sum_{(k, h) \in\left[\mathbb{Z}_{i j}\right]_{m}} a_{(i-k)(j-h)}\left[a_{k h}\right]_{m-1}, m \leq i+j \leq m n, m \geq 2,
\end{array}\right.
$$

in which the family $\left[\mathbb{Z}_{i j}\right]_{m}$ is given by

$$
\begin{gathered}
\left\{(k, h) \in \mathbb{Z}_{+}^{2}:(k, h) \leq(i, j), 1 \leq i-k+j-h \leq n,\right. \\
m-1 \leq k+h \leq(m-1) n\} .
\end{gathered}
$$

Proof. In the case of $m=1$, it is clear that (2.2) holds with respect to $\left[a_{i j}\right]_{1}=a_{i j}, 1 \leq i+j \leq n$. When $m \geq 2$, by putting

$$
f(\varepsilon, \delta)=\left(\sum_{1 \leq i+j \leq n} a_{i j} \varepsilon^{i} \delta^{j}\right)^{m}
$$

we have the Maclaurin formula of $f$ up to order $m n$ as follows

$$
\begin{aligned}
f(\varepsilon, \delta)= & \sum_{0 \leq i+j \leq m n} \frac{1}{i ! j !} \frac{\partial^{i+j} f}{\partial \varepsilon^{i} \partial \delta^{j}}(0,0) \varepsilon^{i} \delta^{j} \\
& =\sum_{m \leq i+j \leq m n} \frac{1}{i ! j !} \frac{\partial^{i+j} f}{\partial \varepsilon^{i} \partial \delta^{j}}(0,0) \varepsilon^{i} \delta^{j} .
\end{aligned}
$$

Therefore, we deduce from (2.2) and (2.5), that

$$
\left[a_{i j}\right]_{m}=\frac{1}{i ! j !} \frac{\partial^{i+j} f}{\partial \varepsilon^{i} \partial \delta^{j}}(0,0), m \leq i+j \leq m n .
$$

Moreover, from (2.2) and (2.4), $f$ can be rewritten as follows

$$
\left\{\begin{array}{l}
f(\varepsilon, \delta)=f_{1}(\varepsilon, \delta) f_{2}(\varepsilon, \delta), \\
f_{1}(\varepsilon, \delta)=\left(\sum_{1 \leq i+j \leq n} a_{i j} \varepsilon^{i} \delta^{j}\right), \\
f_{2}(\varepsilon, \delta)=\left(\sum_{m-1 \leq i+j \leq(m-1) n}\left[a_{i j}\right]_{m-1} \varepsilon^{i} \delta^{j}\right) .
\end{array}\right.
$$

Since $f$ is differentiable at $(0,0)$, it follows from $(2.7)$, that

$$
\frac{\partial^{i+j} f}{\partial \varepsilon^{i} \partial \delta^{j}}(0,0)=\sum_{(k, h) \leq(i, j)} C_{(i, j)}^{(k, h)} \frac{\partial^{i-k+j-h} f_{1}}{\partial \varepsilon^{i-k} \partial \delta^{j-h}}(0,0) \frac{\partial^{k+h} f_{2}}{\partial \varepsilon^{k} \partial \delta^{h}}(0,0) .
$$

Since $(2.2),(2.7)_{2}$ and $\left[a_{i j}\right]_{1}=a_{i j}$, we obtain

$$
a_{i j}=\frac{1}{i ! j !} \frac{\partial^{i+j} f_{1}}{\partial \varepsilon^{i} \partial \delta^{j}}(0,0), 1 \leq i+j \leq n .
$$


Hence, we have

$$
\frac{\partial^{i-k+j-h} f_{1}}{\partial \varepsilon^{i-k} \partial \delta^{j-h}}(0,0)=(i-k) !(j-h) ! a_{(i-k)(j-h)}, 1 \leq i-k+j-h \leq n .
$$

Moreover, we also obtain from $(2.2),(2.6)$ and $(2.7)_{3}$, that

$$
\frac{\partial^{k+h} f_{2}}{\partial \varepsilon^{k} \partial \delta^{h}}(0,0)=k ! h !\left[a_{k h}\right]_{m-1}, m-1 \leq k+h \leq(m-1) n .
$$

On account of $(2.6),(2.8),(2.10)$ and $(2.11)$, we deduce that (2.3) hlods. Thus, the proof of this lemma is complete.

REMARK 1. In [18], we obtained an original result which is more general than Lemma 2. However, since [18] was unpublished, we must give the detailed proof of Lemma 2 in this paper.

\section{Unique solvability and regularity}

In this section, we shortly list some results which were obtained in [15] and can be independently studied by the contracted procedure in [12].

We make some following essential assumptions:

$\left(A_{\mu}\right) \frac{\partial \mu}{\partial t} \in L^{1}\left(0, T ; L^{\infty}(0,1)\right), \mu(x, t) \geq \mu_{0}>0 ;$

$\left(A_{G H}^{(1)}\right) G, H \in L^{2}(\mathbb{R})$;

$\left(A_{G H}^{(2)}\right) \exists K_{G}, K_{H}>0$ :

$$
\begin{gathered}
|G(u)-G(v)| \leq K_{G}|u-v|, \\
|H(u)-H(v)| \leq K_{H}|u-v|,
\end{gathered}
$$

for $u, v \in \mathbb{R}$;

$\left(A_{F}\right) F \in L^{2}((0,1) \times(0, T))$;

$\left(A_{g}\right) g_{0}, g_{1} \in H^{1}(0, T)$;

$\left(A_{k}\right) k_{0}, k_{1} \in W^{1,1}(0, T)$;

$\left(A_{u}\right) u_{0} \in H^{1}(0,1), u_{1} \in L^{2}(0,1)$.

In this paper, we say that a function

$$
u \in H^{1}\left(0, T ; L^{2}(0,1)\right) \cap L^{\infty}\left(0, T ; H^{1}(0,1)\right)
$$

is a weak solution of problem (1.1)-(1.4) iff

$$
\left\{\begin{aligned}
& \frac{d}{d t}\left\langle\frac{\partial u}{\partial t}(t), v\right\rangle+\left\langle\mu(\cdot, t) \frac{\partial u}{\partial x}(t),\right.\left.v^{\prime}\right\rangle+Q_{0}(t) v(0)+Q_{1}(t) v(1) \\
&+\left\langle G(u(t))+H\left(\frac{\partial u}{\partial t}(t)\right), v\right\rangle=\langle F(\cdot, t), v\rangle, \\
& u(x, 0)=u_{0}(x), \frac{\partial u}{\partial t}(x, 0)= u_{1}(x), \\
& Q_{0}(t)=g_{0}(t)+\int_{0}^{t} k_{0}(t-s) u(0, s) d s, \\
& Q_{1}(t)=g_{1}(t)+\int_{0}^{t} k_{1}(t-s) u(1, s) d s,
\end{aligned}\right.
$$

for each $v \in H^{1}(0,1)$ and a.e. time $0 \leq t \leq T$. In this case we can also say that problem (1.1)-(1.4) is weakly solvable in $H^{1}\left(0, T ; L^{2}(0,1)\right) \cap L^{\infty}\left(0, T ; H^{1}(0,1)\right)$.

Then we have the following theorem:

Theorem 1. Let $\left(A_{\mu}\right),\left(A_{G H}^{(1)}\right),\left(A_{G H}^{(2)}\right),\left(A_{F}\right),\left(A_{g}\right),\left(A_{k}\right)$ and $\left(A_{u}\right)$ hold. Then, for $T>0$, problem (1.1)-(1.4) has a unique weak solution $u(x, t)$ satisfying

$$
u \in H^{1}\left(0, T ; L^{2}(0,1)\right) \cap L^{\infty}\left(0, T ; H^{1}(0,1)\right) .
$$


REMARK 2. When $H\left(\frac{\partial u}{\partial t}\right)=\lambda\left|\frac{\partial u}{\partial t}\right|^{p} \frac{\partial u}{\partial t}, p>0, \lambda<0$, it fails to apply FaedoGalerkin method for the unique solvability of problem (1.1)-(1.4). This method is only applicable for linear or some special nonlinear problems (as in [5], [11], [14], $[19]-[25])$.

REMARK 3. For some special nonlinear forms of $G(u)+H\left(\frac{\partial u}{\partial t}\right)$ such as

$$
\begin{gathered}
K|u|^{p} u+\lambda\left|\frac{\partial u}{\partial t}\right|^{q} \frac{\partial u}{\partial t},(p, q) \in \mathbb{R}_{+}^{2} \backslash(0,0),(K, \lambda) \in \mathbb{R}_{+}^{2}, \\
G(u)+K \frac{\partial u}{\partial t}, K \in \mathbb{R},
\end{gathered}
$$

it is possible to apply Faedo-Galerkin approximation for the unique solvability of problem (1.1)-(1.4); however, some more assumptions for $\mu, G$ and $H$ must be modified and unfortunately the proofs in such cases are usually not only very long but also truly messy such as a priori estimates or passing to the limit by monotone techniques as in [16] and [17].

To study the weak solution's smoothness with respect to the smoothness of given data, assumptions $\left(A_{\mu}\right),\left(A_{G H}^{(1)}\right),\left(A_{G H}^{(2)}\right),\left(A_{F}\right),\left(A_{g}\right),\left(A_{k}\right)$ and $\left(A_{u}\right)$ are strengthened as follows:

$$
\begin{aligned}
& \left(\widetilde{A}_{\mu}\right) \mu \in C^{1}([0,1] \times[0, T]), \frac{\partial^{2} \mu}{\partial t^{2}} \in L^{2}\left(0, T ; L^{\infty}(0,1)\right), \mu(x, t) \geq \mu_{0}>0 ; \\
& \left(\widetilde{A}_{G H}\right) G, H \in C^{1}(\mathbb{R}),\left|H^{\prime}(\eta)\right| \leq C_{H}|\eta|^{\alpha}, \forall \eta \in \mathbb{R}, \text { and } \alpha>0, C_{H} \geq 0 ; \\
& \left(\widetilde{A}_{F}\right) F, \frac{\partial F}{\partial t} \in L^{2}((0,1) \times(0, T)) ; \\
& \quad\left(\widetilde{A}_{g}\right) g_{0}, g_{1} \in H^{2}(0, T) ; \\
& \left(\widetilde{A}_{k}\right) k_{0}, k_{1} \in W^{2,1}(0, T) ; \\
& \left(\widetilde{A}_{u}\right) u_{0} \in H^{2}(0,1), u_{1} \in H^{1}(0,1) .
\end{aligned}
$$

It is clear that problem (1.1)-(1.4) has a unique weak solution

$$
u \in H^{1}\left(0, T ; L^{2}(0,1)\right) \cap L^{\infty}\left(0, T ; H^{1}(0,1)\right)
$$

for which $\left(\widetilde{A}_{\mu}\right),\left(\widetilde{A}_{G H}\right),\left(\widetilde{A}_{F}\right),\left(\widetilde{A}_{g}\right),\left(\widetilde{A}_{k}\right)$ and $\left(\widetilde{A}_{u}\right)$ hold. The regularity of this weak solution is stated in the following theorem:

TheOREM 2. Let $\left(\widetilde{A}_{\mu}\right),\left(\widetilde{A}_{G H}\right),\left(\widetilde{A}_{F}\right),\left(\widetilde{A}_{g}\right),\left(\widetilde{A}_{k}\right)$ and $\left(\widetilde{A}_{u}\right)$ hold. Then, for $T>0$, problem (1.1)-(1.4) has a unique weak solution $u(x, t)$ satisfying

$$
u \in H^{2}\left(0, T ; L^{2}(0,1)\right) \cap H^{1}\left(0, T ; H^{1}(0,1)\right) \cap L^{\infty}\left(0, T ; H^{2}(0,1)\right) .
$$

\section{The stability of the weak solution}

In this section, we study the stability of the weak solution of problem (1.1)-(1.4) in the sense that this weak solution continuously depends on some given data.

By assuming the functions $G, H, u_{0}$ and $u_{1}$ satisfy $\left(\widetilde{A}_{G H}\right)$ and $\left(\widetilde{A}_{u}\right)$, we have from Theorem 2 that problem (1.1)-(1.4) has a unique weak solution $u$ depending on $\mu, F, g_{0}, g_{1}, k_{0}$ and $k_{1}$.

Consider

$$
u=u\left(\mu, F, g_{0}, g_{1}, k_{0}, k_{1}\right),
$$

where $\mu, F, g_{0}, g_{1}, k_{0}$ and $k_{1}$, respectively, satisfy $\left(\widetilde{A}_{\mu}\right),\left(\widetilde{A}_{F}\right),\left(\widetilde{A}_{g}\right)$ and $\left(\widetilde{A}_{k}\right)$. Let $G, H, u_{0}$ and $u_{1}$ be fixed functions such that $\left(\widetilde{A}_{G H}\right)$ and $\left(\widetilde{A}_{u}\right)$ hold. For $\mu_{0}>0$ 
given, we put

$$
\begin{array}{r}
\Xi\left(\mu_{0}\right)=\left\{\left(\mu, F, g_{0}, g_{1}, k_{0}, k_{1}\right): \mu, F, g_{0}, g_{1}, k_{0} \text { and } k_{1}\right. \\
\text { satisfy } \left.\left(\widetilde{A}_{\mu}\right),\left(\widetilde{A}_{F}\right),\left(\widetilde{A}_{g}\right) \text { and }\left(\widetilde{A}_{k}\right), \text { respectively }\right\} .
\end{array}
$$

Right then, we have the following theorem:

Theorem 3. Let $\left(\widetilde{A}_{\mu}\right),\left(\widetilde{A}_{G H}\right),\left(\widetilde{A}_{F}\right),\left(\widetilde{A}_{g}\right),\left(\widetilde{A}_{k}\right)$ and $\left(\widetilde{A}_{u}\right)$ hold. For every $T>0$, the weak solution $u(x, t)$ of problem (1.1)-(1.4) is stable with respect to $\mu, F$, $g_{0}, g_{1}, k_{0}$ and $k_{1}$ in the sense that if $\left(\mu, F, g_{0}, g_{1}, k_{0}, k_{1}\right),\left(\mu_{i}, F_{i}, g_{0 i}, g_{1 i}, k_{0 i}, k_{1 i}\right) \in$ $\Xi\left(\mu_{0}\right)$ and

$$
\left\{\begin{array}{lll}
\mu_{i} \rightarrow \mu & \text { in } & C^{1}([0,1] \times[0, T]), \\
F_{i} \rightarrow F, \frac{\partial F_{i}}{\partial t} \rightarrow \frac{\partial F}{\partial t} & \text { in } & L^{2}\left(0, T ; L^{2}(0,1)\right), \\
g_{0 i} \rightarrow g_{0}, g_{1 i} \rightarrow g_{1} & \text { in } & H^{2}(0, T), \\
k_{0 i} \rightarrow k_{0}, k_{1 i} \rightarrow k_{1} & \text { in } & W^{2,1}(0, T),
\end{array}\right.
$$

when $i \rightarrow+\infty$, then

$$
\left\{\begin{array}{lll}
u_{i} \rightarrow u & \text { in } & L^{\infty}\left(0, T ; H^{1}(0,1)\right) \\
\frac{\partial u_{i}}{\partial t} \rightarrow \frac{\partial u}{\partial t} & \text { in } & L^{\infty}\left(0, T ; L^{2}(0,1)\right)
\end{array}\right.
$$

as $i \rightarrow+\infty$, where $u_{i}=u_{i}\left(\mu_{i}, F_{i}, g_{0 i}, g_{1 i}, k_{0 i}, k_{1 i}\right)$.

Proof. From Theorem 2, we can deduce that

$$
\left\{\begin{array}{l}
\left\|\frac{\partial u}{\partial t}(t)\right\|_{L^{2}(0,1)}^{2}+\mu_{0}\left\|\frac{\partial u}{\partial x}(t)\right\|_{L^{2}(0,1)}^{2} \leq M_{T} \\
\left\|\frac{\partial^{2} u}{\partial t^{2}}(t)\right\|_{L^{2}(0,1)}^{2}+\mu_{0}\left\|\frac{\partial^{2} u}{\partial x \partial t}(t)\right\|_{L^{2}(0,1)}^{2} \leq M_{T}
\end{array}\right.
$$

for all $t \in[0, T]$ if the given data $\mu, F, g_{0}, g_{1}, k_{0}$ and $k_{1}$ satisfy

$$
\left\{\begin{array}{l}
\|\mu\|_{C^{1}([0,1] \times[0, T])} \leq \bar{\mu} \\
\|F\|_{L^{2}((0,1) \times(0, T))}+\left\|\frac{\partial F}{\partial t}\right\|_{L^{2}((0,1) \times(0, T))} \leq \bar{F} \\
\left\|g_{0}\right\|_{H^{2}(0, T)}+\left\|g_{1}\right\|_{H^{2}(0, T)} \leq \bar{g} \\
\left\|k_{0}\right\|_{W^{2,1}(0, T)}+\left\|k_{1}\right\|_{W^{2,1}(0, T)} \leq \bar{k}
\end{array}\right.
$$

where $\bar{\mu}, \bar{F}, \bar{g}$ and $\bar{k}$ are fixed positive constants, and $M_{T}$ is a positive constant depending only on $T, \mu_{0}, u_{0}, u_{1}, \bar{\mu}, \bar{F}, \bar{g}, \bar{k}$ but is independent of $\mu, F, g_{0}, g_{1}, k_{0}$, $k_{1}$.

From (4.1), it is clear that there exist positive constants $\bar{\mu}, \bar{F}, \bar{g}$ and $\bar{k}$ such that $\mu_{i}, F_{i}, g_{0 i}, g_{1 i}, k_{0 i}$ and $k_{1 i}$ satisfy (4.4) for $\left(\mu, F, g_{0}, g_{1}, k_{0}, k_{1}\right)=\left(\mu_{i}, F_{i}, g_{0 i}, g_{1 i}, k_{0 i}, k_{1 i}\right)$. Therefore, we conclude that the weak solution $u_{i}(x, t)$ of problem (1.1)-(1.4) with $\left(\mu, F, g_{0}, g_{1}, k_{0}, k_{1}\right)=\left(\mu_{i}, F_{i}, g_{0 i}, g_{1 i}, k_{0 i}, k_{1 i}\right)$ satisfies (4.3), namely we obtain

$$
\left\{\begin{array}{l}
\left\|\frac{\partial u_{i}}{\partial t}(t)\right\|_{L^{2}(0,1)}^{2}+\mu_{0}\left\|\frac{\partial u_{i}}{\partial x}(t)\right\|_{L^{2}(0,1)}^{2} \leq M_{T} \\
\left\|\frac{\partial^{2} u_{i}}{\partial t^{2}}(t)\right\|_{L^{2}(0,1)}^{2}+\mu_{0}\left\|\frac{\partial^{2} u_{i}}{\partial x \partial t}(t)\right\|_{L^{2}(0,1)}^{2} \leq M_{T}
\end{array}\right.
$$

for all $t \in[0, T]$. 
Now, by letting $\widehat{\mu}_{i}, \widehat{F}_{i}, \widehat{g}_{0 i}, \widehat{g}_{1 i}, \widehat{k}_{0 i}$ and $\widehat{k}_{1 i}$ be functions given by

$$
\left\{\begin{array}{l}
\widehat{\mu}_{i}=\mu_{i}-\mu, \\
\widehat{F}_{i}=F_{i}-F, \\
\widehat{g}_{0 i}=g_{0 i}-g_{0}, \widehat{g}_{1 i}=g_{1 i}-g_{1}, \\
\widehat{k}_{0 i}=k_{0 i}-k_{0}, \widehat{k}_{1 i}=k_{1 i}-k_{1},
\end{array}\right.
$$

we deduce that $w_{i}(x, t)=u_{i}(x, t)-u(x, t)$ satisfies the following variational problem:

$$
\left\{\begin{array}{l}
\left\langle\frac{\partial^{2} w_{i}}{\partial t^{2}}(t), v\right\rangle+\left\langle\mu(\cdot, t) \frac{\partial w_{i}}{\partial x}(t), v^{\prime}\right\rangle+\widehat{Q}_{0 i}(t) v(0)+\widehat{Q}_{1 i}(t) v(1) \\
=-\left\langle G\left(u_{i}(t)\right)-G(u(t))+H\left(\frac{\partial u_{i}}{\partial t}(t)\right)-H\left(\frac{\partial u}{\partial t}(t)\right), v\right\rangle \\
\quad-\left\langle\widehat{\mu}_{i}(\cdot, t) \frac{\partial u_{i}}{\partial x}(t), v^{\prime}\right\rangle+\left\langle\widehat{F}_{i}(\cdot, t), v\right\rangle \\
w_{i}(x, 0)=\frac{\partial w_{i}}{\partial t}(x, 0)=0 \\
\widehat{Q}_{0 i}(t)=\check{g}_{0 i}(t)+\int_{0}^{t} k_{0}(t-s) w_{i}(0, s) d s \\
\widehat{Q}_{1 i}(t)=\check{g}_{1 i}(t)+\int_{0}^{t} k_{1}(t-s) w_{i}(1, s) d s \\
\check{g}_{0 i}(t)=\widehat{g}_{0 i}(t)+\int_{0}^{t} \widehat{k}_{0}(t-s) u_{i}(0, s) d s \\
\check{g}_{1 i}(t)=\widehat{g}_{1 i}(t)+\int_{0}^{t} \widehat{k}_{1}(t-s) u_{i}(1, s) d s
\end{array}\right.
$$

for each $v \in H^{1}(0,1)$ and a.e. time $0 \leq t \leq T$.

In (4.7), replacing $v$ by $\frac{\partial w_{i}}{\partial t}$, then integrating from 0 to $t$, we obtain that

$$
\begin{aligned}
\complement_{i}(t)= & \int_{0}^{t}\left\langle\frac{\partial \mu}{\partial s}(\cdot, s),\left|\frac{\partial w_{i}}{\partial x}(s)\right|^{2}\right\rangle d s-2 \int_{0}^{t}\left\langle\widehat{\mu}_{i}(\cdot, s) \frac{\partial u_{i}}{\partial x}(s), \frac{\partial^{2} w_{i}}{\partial x \partial s}(s)\right\rangle d s \\
& -2 \int_{0}^{t}\left\langle G\left(u_{i}(s)\right)-G(u(s)), \frac{\partial w_{i}}{\partial s}(s)\right\rangle d s \\
& -2 \int_{0}^{t}\left\langle H\left(\frac{\partial u_{i}}{\partial s}(s)\right)-H\left(\frac{\partial u}{\partial s}(s)\right), \frac{\partial w_{i}}{\partial s}(s)\right\rangle d s \\
& -2 \int_{0}^{t} \widehat{Q}_{0 i}(s) \frac{\partial w_{i}}{\partial s}(0, s) d s-2 \int_{0}^{t} \widehat{Q}_{1 i}(s) \frac{\partial w_{i}}{\partial s}(1, s) d s \\
& +2 \int_{0}^{t}\left\langle\widehat{F}_{i}(\cdot, s), \frac{\partial w_{i}}{\partial s}(s)\right\rangle d s
\end{aligned}
$$

in which

$$
\subset_{i}(t)=\left\|\frac{\partial w_{i}}{\partial t}(t)\right\|_{L^{2}(0,1)}^{2}+\left\|\sqrt{\mu(\cdot, t)} \frac{\partial w_{i}}{\partial x}(t)\right\|_{L^{2}(0,1)}^{2} .
$$


From $(4.5),(4.8),(4.9)$ and assumptions $\left(\widetilde{A}_{\mu}\right),\left(\widetilde{A}_{G H}\right),\left(\widetilde{A}_{g}\right),\left(\widetilde{A}_{k}\right)$, we have some estimates as follows:

$$
\begin{gathered}
\int_{0}^{t}\left\langle\frac{\partial \mu}{\partial s}(\cdot, s),\left|\frac{\partial w_{i}}{\partial x}(s)\right|^{2}\right\rangle d s \leq \frac{1}{\mu_{0}} \int_{0}^{t}\left\|\frac{\partial \mu}{\partial s}(\cdot, s)\right\|_{L^{\infty}(0,1)} \bigodot_{i}(s) d s \\
-2 \int_{0}^{t}\left\langle\widehat{\mu}_{i}(\cdot, s) \frac{\partial u_{i}}{\partial x}(s), \frac{\partial^{2} w_{i}}{\partial x \partial s}(s)\right\rangle d s \leq \widetilde{M}_{i}+\beta \bigodot_{i}(t)+\int_{0}^{t} \bigodot_{i}(s) d s \\
-2 \int_{0}^{t}\left\langle G\left(u_{i}(s)\right)-G(u(s)), \frac{\partial w_{i}}{\partial s}(s)\right\rangle d s \leq\left(1+T G_{0}^{2}\right) \int_{0}^{t} \bigodot_{i}(s) d s \\
-2 \int_{0}^{t}\left\langle H\left(\frac{\partial u_{i}}{\partial s}(s)\right)-H\left(\frac{\partial u}{\partial s}(s)\right), \frac{\partial w_{i}}{\partial s}(s)\right\rangle d s \\
\left.-2 \int_{0}^{t} \widehat{Q}_{j i}(s) \frac{\partial w_{i}}{\partial s}(j, s) d s \leq \widehat{q}_{j 1 i}+\frac{4 \beta}{\mu_{0}} \bigodot_{i}(t)+\widehat{q}_{j 2 i}\right) \int_{0}^{t} \int_{0}^{t} \bigodot_{i}(s) d s, j=0,1, \\
2 \int_{0}^{t}\left\langle\widehat{F}_{i}(\cdot, s), \frac{\partial w_{i}}{\partial s}(s)\right\rangle d s \leq \int_{0}^{t}\left\|\widehat{F}_{i}(\cdot, s)\right\|_{L^{2}(0,1)}^{2} d s+\int_{0}^{t} \bigodot_{i}(s) d s,
\end{gathered}
$$

for some $\beta>0$ and $\widetilde{M}_{i}, G_{0}, H_{0}, \widehat{q}_{j 1 i}, \widehat{q}_{j 1 i}$ are non-negative constants given by

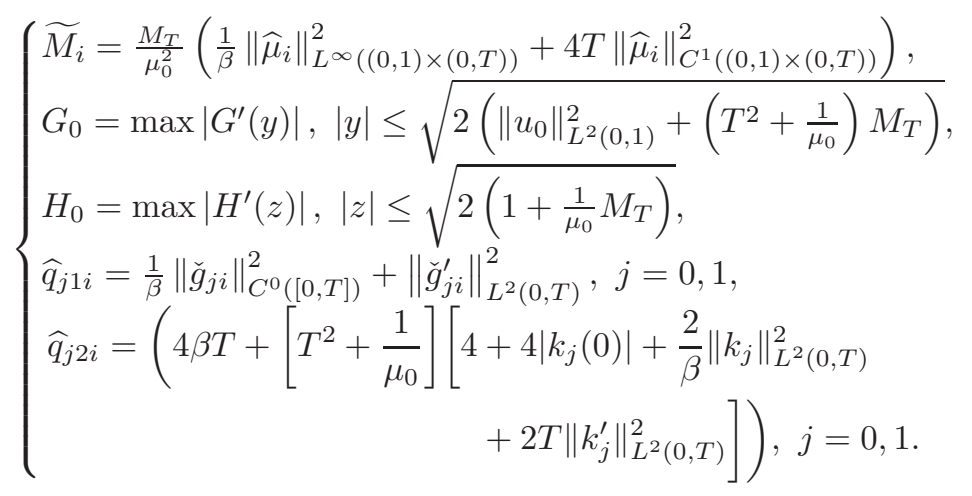

As a result, we deduce from (4.8), (4.10)-(4.16), that

$$
\mathrm{C}_{i}(t) \leq \widehat{M}_{i}+\beta\left(1+\frac{4}{\mu_{0}}\right) \bigodot_{i}(t)+\int_{0}^{t} p_{i}(s) \bigodot_{i}(s) d s,
$$

where $\widehat{M}_{T}$ is a non-negative constant given by

$$
\widehat{M}_{i}=\widetilde{M}_{i}+\widehat{q}_{01 i}+\widehat{q}_{11 i}+\int_{0}^{t}\left\|\widehat{F}_{i}(\cdot, s)\right\|_{L^{2}(0,1)}^{2} d s
$$

and

$$
p_{i}(t)=4+T G_{0}^{2}+H_{0}^{2}+q_{02}+q_{12}+\frac{1}{\mu_{0}}\left\|\frac{\partial \mu}{\partial s}(\cdot, s)\right\|_{L^{\infty}(0,1)} .
$$

Hence, by choosing $\beta>0$ such that $\beta \leq \frac{\mu_{0}}{2\left(\mu_{0}+4\right)}$, we conclude from (4.17), that

$$
\text { C }_{i}(t) \leq 2 \widehat{M}_{i} \exp \left(\int_{0}^{t} p_{i}(s) d s\right) \text { for all } t \in[0, T] .
$$


From $\left(\widetilde{A}_{\mu}\right)$ and $(4.19)$, we have

$$
p_{i} \in L^{1}(0, T), \forall i=1,2, \ldots, \text { and } \exp \left(\int_{0}^{t} p_{i}(s) d s\right)<+\infty .
$$

In addition, on account of $(4.1),(4.7)_{5,6},(4.16)_{1,4}$ and (4.18), we deduce that

$$
\widehat{M}_{i} \rightarrow 0 \text { as } i \rightarrow+\infty \text {. }
$$

Finally, it is clear that (4.2) is obviously concluded from (4.1), (4.9) and (4.20)(4.22). Hence, this proof is complete.

\section{Low-frequency asymptotic expansion of the weak solution}

In this section, let $G(u(x, t)) \equiv \varepsilon G(u(x, t))$ and $H\left(\frac{\partial u}{\partial t}(x, t)\right) \equiv \delta H\left(\frac{\partial u}{\partial t}(x, t)\right)$ such that

$\left(A_{\varepsilon, \delta}\right) \quad \varepsilon, \delta \in \mathbb{R}$.

In addition, we modify smooth assumptions for $G$ and $H$ as follows:

$\left(\widehat{A}_{G H}\right) \quad G, H \in C^{n+1}(\mathbb{R})$ for $n \in \mathbb{N}$ given.

Now we consider the following initial-boundary value problem:

$$
\left\{\begin{array}{l}
\frac{\partial^{2} u}{\partial t^{2}}(x, t)-\frac{\partial}{\partial x}\left(\mu(x, t) \frac{\partial u}{\partial x}(x, t)\right)+\varepsilon G(u(x, t)) \\
\quad+\delta H\left(\frac{\partial u}{\partial t}(x, t)\right)=F(x, t), 0<x<1,0<t<T \\
\mu(0, t) \frac{\partial u}{\partial x}(0, t)=g_{0}(t)+\int_{0}^{t} k_{0}(t-s) u(0, s) d s \\
-\mu(1, t) \frac{\partial u}{\partial x}(1, t)=g_{1}(t)+\int_{0}^{t} k_{1}(t-s) u(1, s) d s \\
u(x, 0)=u_{0}(x), \frac{\partial u}{\partial t}(x, 0)=u_{1}(x)
\end{array}\right.
$$

On account of $\left(\widetilde{A}_{\mu}\right),\left(\widetilde{A}_{G H}\right),\left(A_{\varepsilon, \delta}\right),\left(\widetilde{A}_{F}\right),\left(\widetilde{A}_{g}\right)$ and $\left(\widetilde{A}_{k}\right)$, by Theorem 2 , problem $(5.1)$ has a unique weak solution $u(x, t)$ depending on $(\varepsilon, \delta)$ such that

$$
u=u(\varepsilon, \delta)
$$

satisfying (3.1), namely,

$$
u \in H^{2}\left(0, T ; L^{2}(0,1)\right) \cap H^{1}\left(0, T ; H^{1}(0,1)\right) \cap L^{\infty}\left(0, T ; H^{2}(0,1)\right) .
$$

Our purpose here is to investigate the low-frequency asymptotic expansion (see [9]) of the weak solution $u(x, t)$ of problem (5.1) with respect to two parameters $\varepsilon$, $\delta$.

By putting

$$
\left\{\begin{array}{l}
U u \equiv \frac{\partial^{2} u}{\partial t^{2}}-\frac{\partial}{\partial x}\left(\mu(x, t) \frac{\partial u}{\partial x}\right) \\
\partial U_{0} u \equiv \mu(0, t) \frac{\partial u}{\partial x}(0, t) \\
\partial U_{1} u \equiv-\mu(1, t) \frac{\partial u}{\partial x}(1, t)
\end{array}\right.
$$

we concern the solvable problem $\left(U_{\varepsilon \delta}\right)$ given by

$$
\left\{\begin{array}{l}
U u=-\varepsilon G(u)-\delta H\left(\frac{\partial u}{\partial t}\right)+F(x, t), 0<x<1,0<t<T \\
\partial U_{0} u=g_{0}(t)+\int_{0}^{t} k_{0}(t-s) u(0, s) d s \\
\partial U_{1} u=g_{1}(t)+\int_{0}^{t} k_{1}(t-s) u(1, s) d s \\
u(x, 0)=u_{0}(x), \frac{\partial u}{\partial t}(x, 0)=u_{1}(x) \\
u \equiv u(\varepsilon, \delta) \in H^{2}\left(0, T ; L^{2}(0,1)\right) \cap H^{1}((0, T) \times(0,1)) \cap L^{\infty}\left(0, T ; H^{2}(0,1)\right),
\end{array}\right.
$$


which is perturbed with respect to both small parameters $\varepsilon, \delta$ such that

$$
|\varepsilon| \leq 1, \quad|\delta| \leq 1
$$

Firstly we construct a sequence of weak solutions $\left\{u_{i j}\right\}$ from a family of solvable problems $\left\{\left(U_{i j}\right)\right\}$, respectively, such that $i, j \in \mathbb{N}$ and $i+j \leq n$.

We begin with the case $i=j=0$. Let $u_{00}$ be the weak solution of problem $\left(U_{00}\right)$ in regard to $\varepsilon=\delta=0$, namely we have

$$
\left(U_{00}\right)\left\{\begin{array}{l}
U u_{00} \equiv A_{00} \equiv F(x, t), 0<x<1,0<t<T \\
\partial U_{0} u_{00}=g_{0}(t)+\int_{0}^{t} k_{0}(t-s) u_{00}(0, s) d s \\
\partial U_{1} u_{00}=g_{1}(t)+\int_{0}^{t} k_{1}(t-s) u_{00}(1, s) d s \\
u_{00}(x, 0)=u_{0}(x), \frac{\partial u_{00}}{\partial t}(x, 0)=u_{1}(x) \\
u_{00} \in H^{2}\left(0, T ; L^{2}(0,1)\right) \cap H^{1}((0, T) \times(0,1)) \cap L^{\infty}\left(0, T ; H^{2}(0,1)\right) .
\end{array}\right.
$$

When $1 \leq i+j \leq n$, consider $\left\{u_{i j}\right\}$ as the sequence of the weak solutions of the family of solvable problems $\left\{\left(U_{i j}\right)\right\}$, which is defined as follows

$$
\left(U_{i j}\right)\left\{\begin{array}{l}
U u_{i j} \equiv A_{i j}, 0<x<1,0<t<T \\
\partial U_{0} u_{i j}=\int_{0}^{t} k_{0}(t-s) u_{i j}(0, s) d s \\
\partial U_{1} u_{i j}=\int_{0}^{t} k_{1}(t-s) u_{i j}(1, s) d s \\
u_{i j}(x, 0)=0, \frac{\partial u_{i j}}{\partial t}(x, 0)=0 \\
u_{i j} \in H^{2}\left(0, T ; L^{2}(0,1)\right) \cap H^{1}((0, T) \times(0,1)) \cap L^{\infty}\left(0, T ; H^{2}(0,1)\right),
\end{array}\right.
$$

where $A_{i j}, 1 \leq i+j \leq n$, are defined by the following recursive scheme:

$$
\left\{\begin{array}{l}
A_{10}=-G\left(u_{00}\right) \\
A_{01}=-H\left(\frac{\partial u_{00}}{\partial t}\right) \\
A_{i 0}=-\sum_{k=1}^{i+j-1} \frac{1}{k !} G^{(k)}\left(u_{00}\right)\left[u_{(i-1) 0}\right]_{k}, 2 \leq i \leq n \\
A_{0 j}=-\sum_{k=1}^{i+j-1} \frac{1}{k !} H^{(k)}\left(\frac{\partial u_{00}}{\partial t}\right)\left[\frac{\partial u_{0(j-1)}}{\partial t}\right]_{k}, 2 \leq j \leq n \\
A_{i j}=-\sum_{k=1}^{i+j-1} \frac{1}{k !}\left(G^{(k)}\left(u_{00}\right)\left[u_{(i-1) j}\right]_{k}-H^{(k)}\left(\frac{\partial u_{00}}{\partial t}\right)\left[\frac{\partial u_{i(j-1)}}{\partial t}\right]_{k}\right) \\
\quad 2 \leq i+j \leq n, i \geq 1, j \geq 1
\end{array}\right.
$$

in which $\left[a_{i j}\right]_{k}$ is defined as what in Lemma 2 .

Then we obtain low-frequency asymptotic expansion of the weak solution $u(x, t)$ of problem (5.1) with respect to two parameters $\varepsilon, \delta$ in the following theorem:

TheOREM 4. For $\varepsilon, \delta \in \mathbb{R}$, if $\left(\widetilde{A}_{\mu}\right),\left(\widetilde{A}_{G H}\right),\left(\widehat{A}_{G H}\right),\left(\widetilde{A}_{F}\right),\left(\widetilde{A}_{g}\right),\left(\widetilde{A}_{k}\right)$ and (5.3) hold, then problem $\left(U_{\varepsilon \delta}\right)$ has a unique weak solution $u=u(\varepsilon, \delta)$ satisfying the asymptotic estimates with respect to two parameters $\varepsilon$ and $\delta$ up to order $n+1$ as 
follows:

$$
\begin{array}{r}
\left\|\frac{\partial u}{\partial t}-\sum_{0 \leq i+j \leq n} \frac{\partial u_{i j}}{\partial t} \varepsilon^{i} \delta^{j}\right\|_{L^{\infty}\left(0, T ; L^{2}(0,1)\right)}+\left\|u-\sum_{0 \leq i+j \leq n} u_{i j} \varepsilon^{i} \delta^{j}\right\|_{L^{\infty}\left(0, T ; H^{1}(0,1)\right)} \\
\leq C\left(\varepsilon^{2}+\delta^{2}\right)^{\frac{n+1}{2}},
\end{array}
$$

in which $u_{i j}$ is the unique weak solution of problem $\left(U_{i j}\right)$, respectively, for $0 \leq$ $i+j \leq n$ and $C$ is a positive constant independent of $\varepsilon, \delta$.

Proof. By putting

$$
\left\{\begin{array}{l}
v \equiv \sum_{i+j \leq n} u_{i j} \varepsilon^{i} \delta^{j} \\
w \equiv u-v
\end{array}\right.
$$

we have that $w$ satisfies the following problem

$$
\left\{\begin{aligned}
& U w=-\varepsilon(G(w+v)-G(v))-\delta\left(H\left(\frac{\partial}{\partial t}(w+v)\right)-H\left(\frac{\partial v}{\partial t}\right)\right) \\
& \quad+W_{n}(\varepsilon, \delta), 0<x<1,0<t<T \\
& \partial U_{0} w=\int_{0}^{t} k_{0}(t-s) w(0, s) d s \\
& \partial U_{1} w=\int_{0}^{t} k_{1}(t-s) w(1, s) d s \\
& w(x, 0)=0, \frac{\partial w}{\partial t}(x, 0)=0 \\
& w \in H^{2}\left(0, T ; L^{2}(0,1)\right) \cap H^{1}((0, T) \times(0,1)) \cap L^{\infty}\left(0, T ; H^{2}(0,1)\right)
\end{aligned}\right.
$$

where

$$
W_{n}(\varepsilon, \delta)=F(x, t)-\varepsilon G(v)-\delta H\left(\frac{\partial v}{\partial t}\right)-\sum_{i+j \leq n} A_{i j} \varepsilon^{i} \delta^{j}
$$

The estimate of $W_{n}(\varepsilon, \delta)$ is given in the following lemma:

Lemma 3. Under assumptions $\left(\widetilde{A}_{\mu}\right),\left(\widetilde{A}_{G H}\right),\left(\widehat{A}_{G H)},\left(\widetilde{A}_{F}\right),\left(\widetilde{A}_{g}\right)\right.$ and $\left(\widetilde{A}_{k}\right)$, the following estimate

$$
\left\|W_{n}(\varepsilon, \delta)\right\|_{L^{\infty}\left(0, T ; L^{2}(0,1)\right)} \leq W_{T}\left(\varepsilon^{2}+\delta^{2}\right)^{\frac{n+1}{2}}
$$

holds for $\varepsilon, \delta \in \mathbb{R}$ satisfying (5.3), where $W_{T}$ is a non-negative constant depending only on $G, H, u_{i j}$ and $\frac{\partial u_{i j}}{\partial t}$ for $0 \leq i+j \leq n$.

The detailed proof of this lemma will be specified later. 
Now, by multiplying the two sides of $(5.9)_{1}$ by $\frac{\partial w}{\partial t}$ and taking into account (5.2), we deduce after integrating with respect to time variable that

$$
\begin{aligned}
\Theta(t)= & \int_{0}^{t}\left\langle\frac{\partial \mu}{\partial s}(\cdot, s),\left(\frac{\partial w}{\partial x}(s)\right)^{2}\right\rangle d s \\
& -2 \int_{0}^{t} \frac{\partial w}{\partial s}(0, s)\left(\int_{0}^{s} k_{0}(s-\tau) w(0, \tau) d \tau\right) d s \\
& -2 \int_{0}^{t} \frac{\partial w}{\partial s}(1, s)\left(\int_{0}^{s} k_{1}(s-\tau) w(1, \tau) d \tau\right) d s \\
& -2 \varepsilon \int_{0}^{t}\left\langle G(w+v)-G(v), \frac{\partial w}{\partial s}(s)\right\rangle d s \\
& -2 \delta \int_{0}^{t}\left\langle H\left(\frac{\partial}{\partial t}(w+v)\right)-H\left(\frac{\partial v}{\partial t}\right), \frac{\partial w}{\partial s}(s)\right\rangle d s \\
& +2 \int_{0}^{t}\left\langle W_{n}(\varepsilon, \delta), \frac{\partial w}{\partial s}(s)\right\rangle d s
\end{aligned}
$$

where

$$
\Theta(t)=\left\|\frac{\partial w}{\partial t}(t)\right\|_{L^{2}(0,1)}^{2}+\left\|\sqrt{\mu(t)} \frac{\partial w}{\partial x}(t)\right\|_{L^{2}(0,1)}^{2} .
$$

From $(2.1),\left(\widetilde{A}_{\mu}\right),\left(\widetilde{A}_{g}\right)$ and $\left(\widetilde{A}_{k}\right)$, also taking into account (5.12) and (5.13), we deduce that

$$
\begin{gathered}
\int_{0}^{t}\left\langle\frac{\partial \mu}{\partial s}(\cdot, s),\left(\frac{\partial w}{\partial x}(s)\right)^{2}\right\rangle \\
\leq \frac{1}{\mu_{0}} \int_{0}^{t}\left\|\frac{\partial \mu}{\partial s}(\cdot, s)\right\|_{L^{\infty}(0,1)} \Theta(s) d s \\
-2 \int_{0}^{t} \frac{\partial w}{\partial s}(\nu, s)\left(\int_{0}^{s} k_{\nu}(s-\tau) w(\nu, \tau) d \tau\right) d s \leq \frac{2 \varrho}{\mu_{0}} \Theta(t)+\bar{k}_{\nu} \int_{0}^{t} \Theta(s) d s
\end{gathered}
$$

in which $\varrho>0$ and $\bar{k}_{\nu}, \nu=0,1$, are non-negative constants defined as follows:

$$
\bar{k}_{\nu}=\left(T^{2}+\frac{1}{\mu_{0}}\right)\left(2+2 \varrho T+4\left|k_{\nu}(0)\right|+\frac{2}{\varrho}\left\|k_{\nu}\right\|_{L^{2}(0, T)}^{2}+2 T\left\|k_{\nu}^{\prime}\right\|_{L^{2}(0, T)}^{2}\right) .
$$

In addition, also regarding (5.12) and (5.13), we obtain from $(5.3),(5.8)$ and $\left(\widetilde{A}_{G H}\right)$, that

$$
\left\{\begin{array}{l}
-2 \varepsilon \int_{0}^{t}\left\langle G(w+v)-G(v), \frac{\partial w}{\partial s}(s)\right\rangle d s \leq \bar{G} \int_{0}^{t} \Theta(s) d s \\
-2 \delta \int_{0}^{t}\left\langle H\left(\frac{\partial}{\partial t}(w+v)\right)-H\left(\frac{\partial v}{\partial t}\right), \frac{\partial w}{\partial s}(s)\right\rangle d s \leq \bar{H} \int_{0}^{t} \Theta(s) d s
\end{array}\right.
$$


for non-negative constants $\bar{G}$ and $\bar{H}$ given by

$$
\left\{\begin{array}{l}
\bar{G}=1+T^{2} \sup \left|G^{\prime}(\eta)\right|^{2} \\
\bar{H}=1+\sup \left|H^{\prime}(\sigma)\right|^{2} \\
|\eta| \leq \sqrt{2}\left(\|u\|_{L^{\infty}\left(0, T ; H^{1}(0,1)\right)}+\sum_{0 \leq i+j \leq n}\left\|u_{i j}\right\|_{L^{\infty}\left(0, T ; H^{1}(0,1)\right)} 2^{i+j}\right) \\
|\sigma| \leq \sqrt{2}\left(\|u\|_{L^{\infty}\left(0, T ; H^{2}(0,1)\right)}+\sum_{0 \leq i+j \leq n}\left\|u_{i j}\right\|_{L^{\infty}\left(0, T ; H^{2}(0,1)\right)} 2^{i+j}\right) .
\end{array}\right.
$$

From Lemma 3 and (5.13), it obviously follows that

$$
2 \int_{0}^{t}\left\langle W_{n}(\varepsilon, \delta), \frac{\partial w}{\partial s}(s)\right\rangle d s \leq T W_{T}\left(\varepsilon^{2}+\delta^{2}\right)^{\frac{n+1}{2}}+\int_{0}^{t} \Theta(s) d s .
$$

Combining (5.12)-(5.19) by choosing $\varrho \in\left(0, \frac{\mu_{0}}{4}\right)$, we conclude that

$$
\frac{\Theta(t)}{\min \left\{1, \mu_{0}\right\}} \leq C\left(\varepsilon^{2}+\delta^{2}\right)^{\frac{n+1}{2}}, \text { for all } t \in[0, T]
$$

for

$$
C \equiv \frac{2 T W_{T}}{\min \left\{1, \mu_{0}\right\}}\left[\exp \left(1+\bar{G}+\bar{H}+\bar{k}_{\nu}+\frac{1}{\mu_{0}} \int_{0}^{t}\left\|\frac{\partial \mu}{\partial s}(\cdot, s)\right\|_{L^{\infty}(0,1)} d s\right)\right]^{2} .
$$

Finally, it is clear that (5.7) is deduced from (5.13), (5.20) and (5.21). Hence, the proof is complete.

\section{Appendix: The detailed proof of Lemma 3}

Recall Lemma 3 in the previous section as follows:

Lemma 3. Under assumptions $\left(\widetilde{A}_{\mu}\right),\left(\widetilde{A}_{G H}\right),\left(\widehat{A}_{G H}\right),\left(\widetilde{A}_{F}\right),\left(\widetilde{A}_{g}\right)$ and $\left(\widetilde{A}_{k}\right)$, the following estimate

$$
\left\|W_{n}(\varepsilon, \delta)\right\|_{L^{\infty}\left(0, T ; L^{2}(0,1)\right)} \leq W_{T}\left(\varepsilon^{2}+\delta^{2}\right)^{\frac{n+1}{2}}
$$

holds for $\varepsilon, \delta \in \mathbb{R}$ satisfying (5.3), where $W_{T}$ is a non-negative constant depending only on $G, H, u_{i j}$ and $\frac{\partial u_{i j}}{\partial t}$ for $0 \leq i+j \leq n$.

Proof. In the cases of $n=0$ and $n=1$, the proof of Lemma 3 is easy, hence we omit the details, here we mainly prove this lemma for $n \geq 2$.

By putting

$$
\widetilde{v} \equiv \sum_{1 \leq i+j \leq n} u_{i j} \varepsilon^{i} \delta^{j},
$$

we have that Taylor's formulas of the functions $G(v)=G\left(u_{00}+\widetilde{v}\right), H\left(\frac{\partial v}{\partial t}\right)=$ $H\left(\frac{\partial}{\partial t}\left(u_{00}+\widetilde{v}\right)\right)$ about the points $u_{00}, \frac{\partial u_{00}}{\partial t}$, respectively, up to order $n$ are given by

$$
\left\{\begin{array}{l}
G(v)=G\left(u_{00}\right)+\sum_{k=1}^{n-1} \frac{1}{k !} G^{(k)}\left(u_{00}\right) \widetilde{v}^{k}+\frac{1}{n !} G^{(n)}\left(u_{00}+\xi \widetilde{v}\right) \widetilde{v}^{n}, \\
H\left(\frac{\partial v}{\partial t}\right)=H\left(\frac{\partial u_{00}}{\partial t}\right)+\sum_{k=1}^{n-1} \frac{1}{k !} H^{(k)}\left(\frac{\partial u_{00}}{\partial t}\right)\left(\frac{\partial \widetilde{v}}{\partial t}\right)^{k}+\frac{1}{n !} H^{(n)}\left(\frac{\partial}{\partial t}\left(u_{00}+\zeta \widetilde{v}\right)\right)\left(\frac{\partial \widetilde{v}}{\partial t}\right)^{n},
\end{array}\right.
$$


where $\xi$, and $\zeta \in(0,1)$. Applying Lemma 2, we deduce from (6.3), that

$$
\left\{\begin{aligned}
G(v)=G\left(u_{00}\right) & +\sum_{k=1}^{n-1} \frac{1}{k !} G^{(k)}\left(u_{00}\right) \sum_{k \leq i+j \leq k n}\left[u_{i j}\right]_{k} \varepsilon^{i} \delta^{j} \\
& +\frac{1}{n !} G^{(n)}\left(u_{00}+\xi \widetilde{v}\right) \sum_{n \leq i+j \leq n^{2}}\left[u_{i j}\right]_{n} \varepsilon^{i} \delta^{j} \\
H\left(\frac{\partial v}{\partial t}\right)=H & \left(\frac{\partial u_{00}}{\partial t}\right)+\sum_{k=1}^{n-1} \frac{1}{k !} H^{(k)}\left(\frac{\partial u_{00}}{\partial t}\right) \sum_{k \leq i+j \leq k n}\left[\frac{\partial u_{i j}}{\partial t}\right]_{k} \varepsilon^{i} \delta^{j} \\
+ & \frac{1}{n !} H^{(n)}\left(\frac{\partial}{\partial t}\left(u_{00}+\zeta \widetilde{v}\right)\right) \sum_{n \leq i+j \leq n^{2}}\left[\frac{\partial u_{i j}}{\partial t}\right]_{n} \varepsilon^{i} \delta^{j}
\end{aligned}\right.
$$

in which

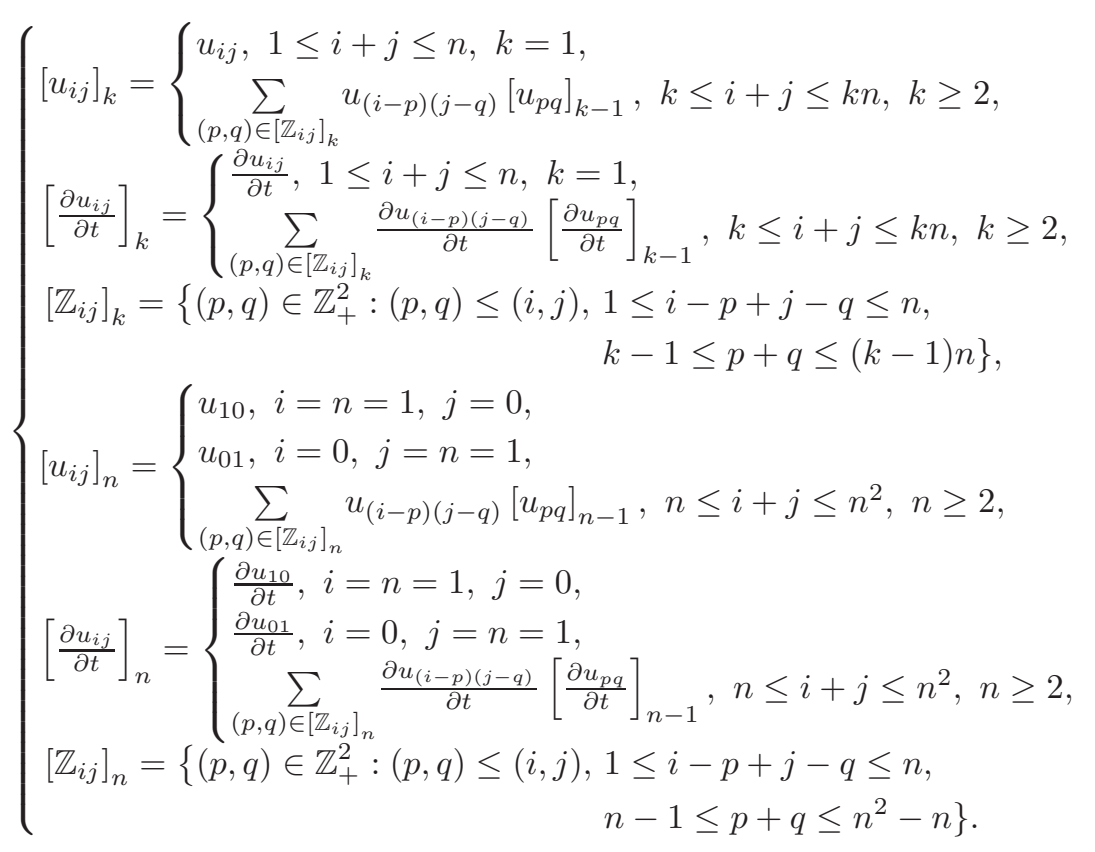

In addition, on account of these identities

$$
\begin{array}{r}
\varepsilon \sum_{k=1}^{n-1} \frac{1}{k !} G^{(k)}\left(u_{00}\right) \sum_{k \leq i+j \leq n-1}\left[u_{i j}\right]_{k} \varepsilon^{i} \delta^{j}=\sum_{1 \leq i+j \leq n-1} \sum_{k=1}^{i+j} \frac{1}{k !} G^{(k)}\left(u_{00}\right)\left[u_{i j}\right]_{k} \varepsilon^{i+1} \delta^{j}, \\
\delta \sum_{k=1}^{n-1} \frac{1}{k !} H^{(k)}\left(\frac{\partial u_{00}}{\partial t}\right) \sum_{k \leq i+j \leq n-1}\left[\frac{\partial u_{i j}}{\partial t}\right]_{k} \varepsilon^{i} \delta^{j} \\
=\sum_{1 \leq i+j \leq n-1} \sum_{k=1}^{i+j} \frac{1}{k !} H^{(k)}\left(\frac{\partial u_{00}}{\partial t}\right)\left[\frac{\partial u_{i j}}{\partial t}\right]_{k} \varepsilon^{i} \delta^{j+1},
\end{array}
$$


we obtain from (6.4), that

$(6.5)$

$$
\left\{\begin{aligned}
& \varepsilon G(v)=\varepsilon G\left(u_{00}\right)+\sum_{2 \leq i+j \leq n, i \geq 1} \sum_{k=1}^{i+j-1} \frac{1}{k !} G^{(k)}\left(u_{00}\right)\left[u_{(i-1) j}\right]_{k} \varepsilon^{i} \delta^{j}+\widetilde{G}(G, \widetilde{v}, \varepsilon, \delta), \\
& \delta H\left(\frac{\partial v}{\partial t}\right)= \delta H\left(\frac{\partial u_{00}}{\partial t}\right)+\sum_{2 \leq i+j \leq n, j \geq 1} \sum_{k=1}^{i+j-1} \frac{1}{k !} H^{(k)}\left(\frac{\partial u_{00}}{\partial t}\right)\left[\frac{\partial u_{i(j-1)}}{\partial t}\right]_{k} \varepsilon^{i} \delta^{j} \\
&+\widetilde{H}\left(H, \frac{\partial \widetilde{v}}{\partial t}, \varepsilon, \delta\right),
\end{aligned}\right.
$$

in which $\widetilde{G}(G, \widetilde{v}, \varepsilon, \delta)$ and $\widetilde{H}\left(H, \frac{\partial \widetilde{v}}{\partial t}, \varepsilon, \delta\right)$ are given as follows

$$
\left\{\begin{aligned}
\widetilde{G}(G, \widetilde{v}, \varepsilon, \delta)= & \varepsilon \sum_{k=1}^{n-1} \frac{1}{k !} G^{(k)}\left(u_{00}\right) \sum_{n \leq i+j \leq k n}\left[u_{i j}\right]_{k} \varepsilon^{i} \delta^{j} \\
& +\frac{\varepsilon}{n !} G^{(n)}\left(u_{00}+\xi \widetilde{v}\right) \sum_{n \leq i+j \leq n^{2}}\left[u_{i j}\right]_{n} \varepsilon^{i} \delta^{j} \\
\widetilde{H}\left(H, \frac{\partial \widetilde{v}}{\partial t}, \varepsilon, \delta\right)= & \delta \sum_{k=1}^{n-1} \frac{1}{k !} H^{(k)}\left(\frac{\partial u_{00}}{\partial t}\right) \sum_{n \leq i+j \leq k n}\left[\frac{\partial u_{i j}}{\partial t}\right]_{k} \varepsilon^{i} \delta^{j} \\
& +\frac{\delta}{n !} H^{(n)}\left(\frac{\partial}{\partial t}\left(u_{00}+\zeta \widetilde{v}\right) \sum_{n \leq i+j \leq n^{2}}\left[\frac{\partial u_{i j}}{\partial t}\right]_{n} \varepsilon^{i} \delta^{j}\right.
\end{aligned}\right.
$$

From (5.10), (6.5) and (6.6), we deduce that

$$
W_{n}(\varepsilon, \delta)=-\widetilde{G}(G, \widetilde{v}, \varepsilon, \delta)-\widetilde{H}\left(H, \frac{\partial \widetilde{v}}{\partial t}, \varepsilon, \delta\right) .
$$

Recall that $u_{i j}, 0 \leq i+j \leq n$, are the weak solutions of problems $\left(U_{i j}\right)$, respectively. From (2.1), (5.3)-(5.5) and (6.2), we deduce that

$$
\left\{\begin{array}{l}
\left|u_{00}(x, t)\right| \leq \sqrt{2}\left\|u_{00}\right\|_{L^{\infty}\left(0, T ; H^{1}(0,1)\right)} \\
\left|u_{00}(x, t)+\xi \widetilde{v}(x, t)\right| \leq \sqrt{2}\left\|u_{00}+\xi \widetilde{v}\right\|_{L^{\infty}\left(0, T ; H^{1}(0,1)\right)}
\end{array}\right.
$$

for $(x, t) \in(0,1) \times(0, T)$ and $\xi \in(0,1)$.

Since $\left(\widehat{A}_{G H}\right)$ and $(6.8)$, it follows that $C_{k}, 1 \leq k \leq n$, such that

$$
\left\{\begin{array}{l}
C_{k} \equiv \sup _{u_{00}}\left|G^{(k)}\left(u_{00}\right)\right| \text { for every } 1 \leq k \leq n-1, \\
C_{n} \equiv \sup _{u_{00}+\xi \widetilde{v}}\left|G^{(n)}\left(u_{00}+\xi \widetilde{v}\right)\right| \xi \in(0,1)
\end{array}\right.
$$

are non-negative constants. Now, combining $(6.6)_{1}$ and (6.9), we conclude that

$$
\|\widetilde{G}(G, \widetilde{v}, \varepsilon, \delta)\|_{L^{\infty}\left(0, T ; L^{2}(0,1)\right)} \leq G_{0}\left(\varepsilon^{2}+\delta^{2}\right)^{\frac{n+1}{2}}
$$

for $G_{0}$ a non-negative constant given by

$$
\begin{aligned}
G_{0}=\sum_{k=1}^{n-1} \sum_{n \leq i+j \leq k n} 2^{\frac{i+j-n}{2}} & \frac{C_{k}}{k !}\left\|\left[u_{i j}\right]_{k}\right\|_{L^{\infty}\left(0, T ; L^{2}(0,1)\right)} \\
& +\frac{C_{n}}{n !} \sum_{n \leq i+j \leq n^{2}} 2^{\frac{i+j-n}{2}}\left\|\left[u_{i j}\right]_{n}\right\|_{L^{\infty}\left(0, T ; L^{2}(0,1)\right)} .
\end{aligned}
$$


Moreover, let $C_{k}^{\prime}, 1 \leq k \leq n$, be non-negative constants defined as follows:

$$
\left\{\begin{array}{l}
C_{k}^{\prime} \equiv \sup _{\frac{\partial u_{00}}{\partial t}}\left|H^{(k)}\left(\frac{\partial u_{00}}{\partial t}\right)\right| \text { for every } 1 \leq k \leq n-1, \\
C_{n}^{\prime} \equiv \sup _{\frac{\partial u_{00}}{\partial t}+\zeta \frac{\partial \widetilde{v}}{\partial t}}\left|H^{(n)}\left(\frac{\partial u_{00}}{\partial t}+\zeta \frac{\partial \widetilde{v}}{\partial t}\right)\right|, \zeta \in(0,1) .
\end{array}\right.
$$

By the same way for the estimate of $\widetilde{G}(G, \widetilde{v}, \varepsilon, \delta)$ in $L^{\infty}\left(0, T ; L^{2}(0,1)\right)$, we also obtain

$$
\|\widetilde{H}(H, \widetilde{v}, \varepsilon, \delta)\|_{L^{\infty}\left(0, T ; L^{2}(0,1)\right)} \leq H_{0}\left(\varepsilon^{2}+\delta^{2}\right)^{\frac{n+1}{2}},
$$

in which $H_{0}$ is a non-negative constant given by

$$
\begin{array}{r}
H_{0}=\sum_{k=1}^{n-1} \sum_{n \leq i+j \leq k n} 2^{\frac{i+j-n}{2}} \frac{C_{k}^{\prime}}{k !}\left\|\left[\frac{\partial u_{i j}}{\partial t}\right]_{k}\right\|_{L^{\infty}\left(0, T ; L^{2}(0,1)\right)} \\
+\frac{C_{n}^{\prime}}{n !} \sum_{n \leq i+j \leq n^{2}} 2^{\frac{i+j-n}{2}}\left\|\left[\frac{\partial u_{i j}}{\partial t}\right]_{n}\right\|_{L^{\infty}\left(0, T ; L^{2}(0,1)\right)}
\end{array} .
$$

Finally, it is clear that (6.10) and (6.13) imply (6.1).

\section{References}

[1] Aassila, M. and Benaissa, A.: Existence of global solutions to a quasi-linear wave equation with general nonlinear damping, Electron. J. Differential Equations (91)(2002), pp. 22.

[2] Dang, A.D. and Alain, P.N.D.: Mixed problem for some semi-linear wave equation with a non-homogeneous condition, Nonlinear Anal. 12(6) (1998) 581-592.

[3] Burton, T.A.: Volterra Integral and Differential Equations, 2d ed., Elsevier B.V., Amsterdam, 2005.

[4] Benaddi, A. and Rao, B.: Energy decay rate of wave equations with indefinite damping, $J$. Differential Equations 161 (2000) 337-357.

[5] Bergounioux, M.; Nguyen, L.T. and Alain, P.N.D.: Mathematical model for a shock problem involving a linear viscoelastic bar, Nonlinear Anal. (13) (2001) 546-561.

[6] Brezis, H.: Analyse fonctionnelle: Théorie et applications, Masson, Paris, 1983.

[7] Collins, P.J.: Differential and Integral Equations, Oxford University Press, 2006.

[8] Evans, L.C.: Partial differential equations, Grad. Stud. Math., Amer. Math. Soc., Vol. 19, 1998.

[9] M.V. Fedoryuk; Partial differential equations $V$ : Asymptotic methods for partial differential equations, Encyclopaedia of mathematical sciences, Springer-Verlag, Vol. 34, 1999.

[10] Lakshmikantham V. and Leela S.: Differential and Integral Inequalities, Academic Press, New York Vol. 1, 1969

[11] Lê, Út V.; Truc, T.T.T. and Nguyen, L.T.: The shock of a rigid body and a linear viscoelastic bar: Global existence and stability of solutions, Proc. the $9^{\text {th }}$ Conf. Sci. Technol. Hcm Univ. Technol. 2005 24-29.

[12] Lê, Út V.: A contracted procedure for the unique solvability of a semilinear wave equation associated with a linear integral equation at the boundary, JP J. Fixed Point Theory and Appl. 3(1) (2008) 49-61.

[13] Lê, Út V.: The well-posedness of a semilinear wave equation associated with a linear integral equation at the boundary, Mem. Differential Equations Math. Phys. 44 (2008) 69-88.

[14] Lê, Út V.; Truc, T.T.T. and Nguyen, L.T.: A shock of a rigid body and a linear viscoelastic bar: Global existence and Stability of the solutions, Vietnam J. Math. Appl. (2008) (accepted).

[15] Lê, Út V.: On a semi-linear wave equation associated with memory conditions at the boundaries: Unique existence and regularity, Dyn. Partial Differ. Equ. (2008) (accepted). 
[16] Lions, J.L.: Quelques méthodes de résolution des problèmes aux limites non-linéaires, DunodGauthier- Villars, Paris, 1969.

[17] Lions, J.L. and Strauss, W.A.: Some nonlinear evolution equations, Bull. Soc. Math. France 93 (1965) 43-96.

[18] Nguyen, L.T.; Lê, Út V. and Truong, L.X.: Mathematical model for a shock problem involving a nonlinear viscoelastic bar, Preprint (2006).

[19] Nguyen, L.T. and Alain, P.N.D.: A semilinear wave equation associated with a linear differential equation with Cauchy data, Nonlinear Anal. 24 (1995) 1261-1279.

[20] Nguyen, L.T.; Alain, P.N.D. and Tran, D.N.: On a shock problem involving a nonlinear viscoelastic bar, Bound. Value Probl. 3 (2005) 337-358.

[21] Nguyen, L.T.; Lê, Út V. and Nguyen, T.T.T.: A shock of a rigid body and a linear viscoelastic bar, Hcm Ped. Univ. J. Natur. Sci. 4(38) (2004) 27-40.

[22] Nguyen, L.T.; Lê, Út V. and Nguyen, T.T.T.: A shock of a rigid body and a linear viscoelastic bar: Global existence and stability of the solutions, Hcm Ped. Univ. J. Natur. Sci. 6(40) (2005) 26-39.

[23] Nguyen, L.T.; Lê, Út V. and Nguyen, T.T.T.: A shock problem involving a linear viscoelastic bar, Nonlinear Anal. 63(2) (2005) 198-224.

[24] Phung, K.D.: Polynomial decay rate for the dissipative wave equation, J. Differential Equations 240 (2007) 92-124.

[25] Santos, M.L.: Asymptotic expansion of solutions to wave equations with a memory condition at the boundary, Electron. J. Differential Equations, 73 (2001), 11 pp.

[26] Sengul, M.T.: An effective method for the existence of the global attractor of a nonlinear wave equation, Appl. Math. E-Notes 7 (2007) 179-185.

[27] Takači, D. and Takači, A: On the approximate solution of a mathematical model of a viscoelastic bar, Nonlinear Anal. 67 (2007) 1560-1569.

[28] Teschl, G.: Nonlinear functional analysis, Lecture Notes in Math., Vienna Univ., Austria, 2001.

[29] Zeidler, E.: Nonlinear functional analysis and its applications, Vol. II, Springer-Verlag, Leipzig, 1989.

[30] Zheng, S: Nonlinear Evolution Equations, Chapman and Hall/CRC, 2004.

Department of Mathematical Sciences, P.O. Box 3000, FI-90014 University of Oulu, FINLAND

E-mail address: ut.van.le@oulu.fi

E-mail address: levanut@gmail.com

E-mail address: utlev@yahoo.com 